\title{
Efectividad de la profilaxis para enfermedad tuberculosa en pacientes infectados por el virus de la inmunodeficiencia humana, Medellín, 2002-2005
}

\author{
María Patricia Arbeláez', Alexánder Arbeláez¹, Rubén Darío Gómez¹, Carlos Rojas", \\ Lázaro Vélez², Sonia Luz Arias', Jorge Nagles ${ }^{3}$, Luz Marina Peláez ${ }^{4}$, \\ Gloria Betancourt ${ }^{5}$, Gloria Velásquez ${ }^{6}$ \\ ${ }^{1}$ Grupo de Epidemiología, Universidad de Antioquia, Medellín, Colombia \\ ${ }^{2}$ Grupo de Enfermedades Infecciosas (Gripe), Universidad de Antioquia, Medellín, Colombia \\ ${ }^{3}$ Clínica las Américas, Medellín, Colombia \\ ${ }^{4}$ Clínica Bolivariana, Medellín, Colombia \\ ${ }^{5}$ Coomeva EPS, Medellín, Colombia \\ ${ }^{6}$ Requiescat in pacem
}

Introducción. La profilaxis para tuberculosis ha sido aceptada mundialmente para prevenir las formas activas de la enfermedad, especialmente, en pacientes positivos para $\mathrm{VIH}$; pero en los países de alta prevalencia es aún controvertida su efectividad y sus indicaciones.

Objetivo. Establecer en pacientes positivos para VIH el nivel de efectividad de dos esquemas de profilaxis contra la tuberculosis: con isoniacida durante nueve meses o pirazinamida/ rifampicina durante 60 días, suministrados en forma autoadministrada, independientemente de la respuesta a la prueba de tuberculina.

Materiales y métodos. Estudio observacional de cohorte. Se conformaron dos grupos, uno con 131 pacientes, quienes voluntariamente aceptaron recibir uno de los dos esquemas profilácticos, si el de pirazinamida/rifampicina no estaba contraindicado. El grupo control estuvo conformado por 200 pacientes seleccionados retrospectivamente, a partir de los registros de un programa de control de pacientes con VIH/sida. El seguimiento para ambos grupos se realizó durante dos años, mediante revisión de la historia clínica.

Resultados. Los grupos no presentaron diferencias estadísticas significativas cuando se compararon sus características clínicas, ni demográficas. Una mayor proporción de pacientes del grupo control tuvieron recuento de CD4<200/ml y carga viral>100.000. En el grupo con profilaxis, $8 \%$ manifestó efectos adversos, una persona presentó tuberculosis $(0,8 \%)$ y en el grupo control $10(5 \%)$ [RR=0,15, IC95\% 0,02-1,18, $p=0,07]$, la protección de la profilaxis fue del $80 \%$, independiente de CD4, carga viral y terapia antirretroviral recibida.

Conclusión. La profilaxis para tuberculosis mostró ser efectiva en pacientes positivos para VIH, independientemente del estado inmune, virológico y el tratamiento antirretroviral recibido.

Palabras clave: tuberculosis/terapia, profilaxis antibiótica, VIH, síndrome de inmunodeficiencia adquirida, terapia antirretroviral altamente activa, efectividad, Colombia.

\section{Effectiveness of prophylaxis against tuberculosis in patients infected with HIV}

Introduction. Prophylaxis against tuberculosis has been recognized as important for preventing clinical forms of tuberculosis, mainly in HIV positive patients. However, in countries with high tuberculosis prevalence, prophylaxis application and effectiveness remains controversial.

Objective. Effectiveness was established for two prophylaxis regimens -isoniazid treatment for nine months and pirazinamid/rifampin for 60 days.

Materials and methods. Two cohort groups of patients diagnosed with HIV/AIDS were compared. One consisted of 131 volunteer patients, who received one of the two prophylactic regimens pirazinamid/rifampin or isoniazid. The tuberculosis treatment drugs were self-administered and independent of tuberculin response tests. The second group consisted of 200 patients selected 
from the records of a HIV/AIDS control program. Follow up for both groups was conducted over a two-year period through clinical records.

Results. The 2 groups were similar with respect to clinical and demographic variables. A higher proportion of patients in the control group had CD4 counts $<200 / \mathrm{ml}$ and viral load $>100,000$ copies. In the prophylactic group, $8 \%$ of patients reported adverse effects due to the drug, and one person had tuberculosis in that group $(0.8 \%)$. Ten persons in the control group contracted tuberculosis $(5 \%)[R R=0.15,95 \% \mathrm{Cl} 0.02-1.18, \mathrm{p}=0.07]$. The prophylaxis protective level was calculated to be $80 \%$, after taking into account CD4, viral load, and effective antiretroviral therapy.

Conclusion. The prophylaxis against tuberculosis was effective in HIV positive patients, independently of the immune status, viral load, and highly effective antiretroviral therapy.

Key words: tuberculosis/therapy, antibiotic prophylaxis, HIV, acquired immunodeficiency syndrome, antiretroviral therapy, highly active, effectiveness, Colombia.

La coinfección entre el virus de inmunodeficiencia humana $(\mathrm{VIH})$ y Mycobacterium tuberculosis es un problema de salud pública creciente en el mundo. Las personas coinfectadas por VIH y $M$. tuberculosis tienen un riesgo mayor de desarrollar tuberculosis activa y los casos de tuberculosis sufren un deterioro mayor de su condición inmunológica, lo cual potencia ambas entidades $(1,2)$.

La pandemia de la infección por el VIH ha contribuido de manera significativa al incremento de la incidencia de tuberculosis. Se estima que cerca de 15 millones de personas están coinfectadas con $M$. tuberculosis y $\mathrm{VIH}$, y casi el $90 \%$ de estas personas se encuentran en países en desarrollo (3). En el mundo el $8 \%$ de la tuberculosis es atribuible al $\mathrm{VIH}-1$ y se teme que esta proporción aumente en el futuro. La tuberculosis continúa siendo la causa de muerte más común en pacientes con sida, afectando a uno de cada tres pacientes (3).

Una vez ocurrida la coinfección por M. tuberculosis y $\mathrm{VIH}$, el riesgo de desarrollar tuberculosis puede aumentar hasta en nueve veces, comparado con las personas seronegativas para $\mathrm{VIH}-1$ (4). El progreso a la forma activa de la enfermedad en personas positivas para $\mathrm{VIH}$, se ha documentado en estudios prospectivos, con tasas de tuberculosis de $5 \%$ a $10 \%$ por año (5).

\section{Correspondencia:}

María Patricia Arbeláez, Facultad Nacional de Salud Pública, Universidad de Antioquia, Calle 62 No. 52-29, piso 2, Medellín Teléfono: (054) 210 6434; fax: (054) 5112506 o 38282 mpam@guajiros.udea.edu.co, patricia.arbelaez@une.net.co

Recibido: 23/04/07; aceptado: 21/08/07
El compromiso extrapulmonar y las presentaciones atípicas de la enfermedad tuberculosa son comunes en formas avanzadas de la infección por VIH, lo que puede dificultar el diagnóstico de la tuberculosis; una vez realizado este diagnóstico, es necesario ajustar la terapia antrirretrovial altamente efectiva, por la toxicidad que puede ocurrir al combinar rifampicina con inhibidores no análogos de la transcriptasa inversa o inhibidores de proteasas; en ocasiones estos ajustes requieren la supervisión de expertos (6). Estudios recientes muestran que, a pesar de la terapia antirretroviral, pueden persistir defectos funcionales de los linfocitos CD4 y, por consiguiente, el riesgo de tuberculosis continúa presente (7).

Las guías de los Centers for Disease Control and Prevention de los Estados Unidos de América (CDC) para la prevención de la tuberculosis en pacientes positivos para $\mathrm{VIH}$, proponen realizar la prueba de tuberculina a los pacientes con recuentos de CD4 menores de 200 células $/ \mathrm{mm}^{3}$; en ausencia de tuberculosis debe suministrarse profilaxis a los pacientes positivos para $\mathrm{VIH}$, si la prueba de tuberculina es superior a $5 \mathrm{~mm}$; sin embargo, la prueba de tuberculina es difícil de interpretar en los casos postivos para VIH pues varios de ellos pueden tener un deterioro avanzado de su sistema inmune y presentar reacciones negativas (anergia), así la infección por tuberculosis esté presente (8).

En Colombia, la Resolución 412, en sus guías para el tratamiento del $\mathrm{VIH}$, se recomienda brindar la profilaxis a toda persona positiva para VIH que resida en áreas de alta transmisión, 
independiente de su respuesta a la prueba de tuberculina, una vez se descarte la enfermedad tuberculosa activa (9).

El presente estudio se propuso establecer el nivel de efectividad de los esquemas de profilaxis conocidos para la prevención de tuberculosis, con isoniacida durante nueve meses o pirazinamida/ rifampicina durante 60 días continuos, suministrados a pacientes positivos para VIH en forma autoadministrada, independientemente del tratamiento antirretroviral y de la respuesta a la prueba de tuberculina.

\section{Materiales y métodos}

Se realizó un estudio observacional de tipo cohorte. Se incluyeron en el estudio sujetos positivos para VIH confirmados por Western blot, mayores de 18 años, sin evidencia clínica de tuberculosis y sin antecedentes de enfermedad tuberculosa entre los años 2002 y 2005, que acudían a programas de control de infección por VIH-sida en Medellín (programa del Seguro Social, de tres aseguradoras privadas y de una clínica privada). Se conformó una cohorte expuesta a profilaxis y otra cohorte retrospectiva de control. El seguimiento se realizó por un periodo promedio de 20 meses.

Para el seguimiento se establecieron dos cohortes:

Grupo con profilaxis: pacientes VIH positivos, que aceptaron participar voluntariamente en el estudio, expuestos a esquemas completos de quimioprofilaxis autoadministrada con isoniacida durante nueve meses o pirazinamida/rifampicina durante 60 días continuos. El esquema de profilaxis con pirazinamida/rifampicina no se asignó a quienes recibían en su terapia antirretrovirales inhibidores de proteasas (10).

Se suministraron dos esquemas autoadministrados de profilaxis:

Esquema con isoniacida: $300 \mathrm{mg} / \mathrm{dia}$ de isoniacida más 50 mg por día de vitamina B6 por 270 días. Se entregó al paciente medicamento suficiente para un mes.

Esquema con pirazinamida/rifampicina: $600 \mathrm{mg}$ por día de rifampicina y $20 \mathrm{mg} / \mathrm{kg}$ por día de pirazinamida que el paciente ingirió en una sola dosis durante 60 días continuos, a partir de entregas mensuales.

En ambos esquemas se instruyó al paciente sobre la necesidad de registrar diariamente el consumo y consultar al servicio cuando se presentara alguna evidencia de reacciones adversas al medicamento.

Grupo control: el grupo control se conformó con pacientes de los programas de control participantes, que no estuvieron expuestos a profilaxis para tuberculosis. La información sobre esta cohorte se obtuvo retrospectivamente a partir de los registros del programa (historia clínica) de los pacientes que consultaron en el segundo semestre del año 2003, periodo para el cual, la cohorte de profilaxis había terminado el esquema.

El tamaño de muestra estimado fue de 115 pacientes por grupo, para una confianza del 0,95, un poder del 0,8 , una relación expuestos a no expuestos de 1:1, una proporción de tuberculosis en no expuestos a quimioprofilaxis de 0,24 y una reducción del riesgo de 0,6 (un riesgo relativo de $0,4)$ utilizando el programa Epi info versión 6.04.

La selección de la muestra en el grupo de profilaxis se hizo mediante invitación de los médicos responsables del programa de control de VIH en las instituciones, entre los pacientes que no tuvieran síntomas compatibles con tuberculosis y que aceptaran participar, previo consentimiento informado. Se excluyeron aquellos pacientes que presentaran alguna de las siguientes condiciones: embarazo, evidencia actual de hepatopatía, tratamiento actual con algún medicamento que tuviera acción micobactericida, contraindicación médica para la profilaxis y antecedente de exposición a quimioprofilaxis antituberculosa. La selección del grupo control se realizó a partir de los registros de consultas de los pacientes durante el segundo semestre del año 2003, de 212 personas que acudieron a control, se revisaron 200 historias clínicas; de las 12 restantes, 10 no estuvieron disponibles y 2 correspondían a menores de 18 años.

El seguimiento de ambos grupos se hizo hasta junio de 2005, para lo cual se revisaron las 
historias clínicas de las visitas que realizaron durante el periodo a la institución de salud donde eran atendidos. Se registraron los datos de la historia clínica en relación con: su estado inmunológico (CD4), carga viral, pruebas sanguíneas (hemoglobina, hematocrito, velocidad de sedimentación globular, linfocitos, transaminasas, fosfatasas alcalinas), infecciones oportunistas, diagnóstico de sida, tiempo de diagnóstico de la infección, terapias recibidas, cumplimiento de la terapia antirretroviral y síntomas clínicos compatibles con tuberculosis. En el grupo que recibió la profilaxis, se aplicó previamente la prueba de tuberculina. Para el grupo control no se contó con esta prueba.

Se efectuó un control de la calidad del dato, comparando los registros de las historias clínicas con las bases de datos, llevado a cabo por una persona fuera del proyecto.

\section{Análisis estadístico}

Se valoró la presencia de la tuberculosis como variable dependiente y la exposición a quimioprofilaxis como variable explicativa; se consideraron covariables el estrato socieconómico, el valor de los linfocitos CD4, la carga viral y la velocidad sedimentación globular para la presentación de la enfermedad. Se estimó el riesgo relativo (RR), con sus respectivos intervalos de confianza, como medida de asociación, comparando las proporciones de incidencia de la enfermedad entre quienes recibieron la profilaxis antibiótica y quienes no la recibieron. El cálculo de la efectividad de la profilaxis se realizó utilizando las fórmulas para dicho fin (1-riesgo relativo $\times 100)(11)$.

Se estableció un nivel de significación para una $p<0,05$, en todos los análisis utilizando la prueba de ji al cuadrado, con las respectivas correcciones de Yates y Fisher. Se compararon las curvas de supervivencia para el desarrollo de la tuberculosis en ambos grupos, utilizando el método de Kaplan Meier y la prueba de Log Rank. Para el ajuste por las distintas variables de confusión, se realizó un análisis multivariado mediante el modelo de regresión logística; si bien la regresión de Cox es la técnica indicada para este tipo de análisis, dada la magnitud de los errores estándar de las estimaciones realizadas, se prefirió presentar los resultados con el modelo logístico, por haber presentado un mejor ajuste con los datos del estudio. En el análisis estadístico se utilizaron los programas Epi Info versión 6.04 y SPSS 11.5 (Chicago, IL).

\section{Resultados}

En el grupo de profilaxis aceptaron participar en el estudio, 162 personas, de las cuales, 31 no hicieron parte de la cohorte, por los siguientes motivos: 13 manifestaron no querer continuar con el tratamiento durante el primer mes, cuatro por sospecha de ser sintomáticos respiratorios al ingreso, dos fallecieron por sida avanzado en el primer mes, siete por presentar los siguientes síntomas: cinco manifestaron náuseas, temblor en manos y gastritis, uno presentó cefalea y otro por eritema en piel, al inicio de la profilaxis; dos por temor a las consecuencias de los medicamentos y tres no acudieron a la primera visita para el suministro de la profilaxis.

Para el grupo control se identificaron 212 pacientes que consultaron al programa para el tratamiento del VIH en una de las instituciones que participaron en el estudio, durante el segundo semestre de 2003. Finalmente, el grupo estuvo constituido por 200 sujetos, ya que para 12 de ellos, 2 eran menores de 18 años y en los 10 restantes, no se encontraron las historias clínicas.

La cohorte expuesta a profilaxis estuvo conformada por 131 personas; 84 (64\%) de ellas recibieron isoniacida y $47(35,9 \%)$ recibieron pirazinamida/rifampicina (cuadro 1). El tiempo de seguimiento de las cohortes fue de 20,8 meses (desviación estándar $=5,04$ ) para el grupo profilaxis y de 21,1 meses (desviación estándar=4,6) para el grupo control. En promedio, a cada paciente se le hicieron 14,1 evaluaciones en el grupo expuesto a profilaxis y 13 en el grupo control.

Los 47 pacientes que recibieron profilaxis con pirazinamida/rifampicina habían terminado su esquema, cuando se alertó del riesgo de muerte por daño hepático grave en quienes recibían inhibidores de proteasa y esta clase de profilaxis (10). A partir de este momento sólo se ofreció la profilaxis con isoniacida. 
Cuadro 1. Características demográficas de los grupos de estudio y control al momento de ingreso al estudio, Medellín, Colombia, 2002-2005.

\begin{tabular}{|c|c|c|c|c|c|c|}
\hline \multicolumn{2}{|c|}{ Características } & \multicolumn{2}{|c|}{$\begin{array}{l}\text { Grupo profilaxis } \\
(n=131)\end{array}$} & \multicolumn{2}{|c|}{$\begin{array}{l}\text { Grupo control } \\
(n=200)\end{array}$} & \multirow[t]{2}{*}{ Valor $\mathbf{P}$} \\
\hline & & Recuento & $\%$ & Recuento & $\%$ & \\
\hline \multirow[t]{2}{*}{ Sexo } & Masculino & 113 & 86,3 & 163 & 81,5 & 0,25 \\
\hline & Femenino & 18 & 13,7 & 37 & 18,5 & 0,25 \\
\hline Edad & $\begin{array}{l}\text { Media } \\
\text { (Desviación estándar) }\end{array}$ & $\begin{array}{c}43,2 \\
8,6\end{array}$ & & $\begin{array}{c}43,2 \\
9,3\end{array}$ & & 0,53 \\
\hline \multicolumn{7}{|c|}{ Nivel socioeconómico } \\
\hline & Alto $(6)$ & 12 & 9,2 & 5 & 2,5 & $0,01^{*}$ \\
\hline & Medio $(3,4,5)$ & 78 & 60,0 & 113 & 56,5 & 0,58 \\
\hline & Bajo $(1,2)$ & 28 & 21,4 & 46 & 23,9 & 0,57 \\
\hline & Sin dato & 13 & 9,9 & 36 & 19 & 0,25 \\
\hline \multicolumn{7}{|c|}{ Clasificación CDC } \\
\hline & $\mathrm{A} 1$ & 28 & 21,4 & 43 & 21,5 & 0,97 \\
\hline & A2 & 28 & 21,4 & 59 & 29,5 & 0,10 \\
\hline & A3 & 13 & 9,9 & 13 & 6,5 & 0,25 \\
\hline & B1 & 2 & 1,5 & 3 & 1,5 & 0,65 \\
\hline & B2 & 12 & 9,2 & 6 & 3 & 0,15 \\
\hline & B3 & 6 & 4,6 & 2 & 1 & 0,09 \\
\hline & $\mathrm{C} 1$ & 1 & 0,8 & 1 & 0,5 & 0,10 \\
\hline & $\mathrm{C} 2$ & 2 & 1,5 & 6 & 3 & 0,62 \\
\hline & C3 & 27 & 20,6 & 41 & 20,5 & 0,98 \\
\hline & Sin dato & 12 & 9,2 & 26 & 13 & 0,28 \\
\hline \multicolumn{7}{|c|}{ Recibe terapia antirretroviral } \\
\hline & Sí & 110 & 84 & 177 & 88,5 & 0,24 \\
\hline & No & 21 & 16 & 23 & 11,5 & 0,23 \\
\hline \multicolumn{7}{|c|}{ CD4 Inicio } \\
\hline & $<200$ & 19 & 14,5 & 47 & 23,5 & $0,04^{*}$ \\
\hline & Entre 200 y 350 & 24 & 18,3 & 39 & 19,5 & 0,78 \\
\hline & $>350$ & 72 & 55 & 96 & 48 & 0,21 \\
\hline & Sin dato & 16 & 12,2 & 18 & 9 & 0,34 \\
\hline \multicolumn{7}{|c|}{ Carga viral (copias/ml) al inicio } \\
\hline & $<3.000$ & 79 & 60,3 & 90 & 45 & $0,01^{*}$ \\
\hline & Entre 3.000 y 10.000 & 8 & 6,1 & 12 & 6 & 0,96 \\
\hline & Entre 10.000 y 30.000 & 4 & 3,1 & 18 & 9 & $0,03^{*}$ \\
\hline & Entre 30.000 y 100.000 & 14 & 10,7 & 30 & 15 & 0,25 \\
\hline & $>100.000$ & 5 & 3,8 & 28 & 14 & $0,01^{*}$ \\
\hline & Sin dato & 21 & 16 & 22 & 11 & 0,18 \\
\hline
\end{tabular}

${ }^{*} p<0,05$

Al momento de iniciar la observación, las cohortes fueron muy similares (cuadro 1).

No se encontraron diferencias por sexo ni edad. El nivel económico de los participantes se clasificó según los niveles de estrato socioeconómico utilizados en Medellín para el período (1 estrato más bajo y 6 estrato más alto); en ambos grupos predominó el estrato medio (estratos 3,4 y 5). Sin embargo, la proporción de personas del estrato alto fue significativamente mayor en el grupo de profilaxis. Tampoco se encontraron diferencias entre los grupos, al comparar el compromiso inmunológico de los pacientes al momento del diagnóstico de $\mathrm{VIH}$, a excepción de los que presentaron un recuento menor de 200 CD4, cuya proporción fue mayor en el grupo control. Según la clasificación de los CDC de 1993, los estadios asintomáticos $\mathrm{A} 1$ y $\mathrm{A} 2$ predominaron en ambos grupos. El mayor compromiso inmune (C3) se observó de forma similar, en $20,6 \%$ del grupo expuesto a profilaxis y en $20,5 \%$ del grupo control (cuadro 1). 
Tampoco hubo diferencias entre las dos cohortes en relación con la exposición a la terapia antirretroviral al momento del ingreso al estudio. De los 331 casos estudiados, 13,3\% no recibía antirretrovirales.

La carga viral reportada al ingreso del estudio se clasificó según el riesgo de progresión a sida. La replicación viral mayor de 100.000 copias $/ \mathrm{ml}$ fue también significativamente más frecuente en el grupo control.

Entre los pacientes que recibieron profilaxis, la reacción a la prueba de tuberculina fue positiva (mayor de $5 \mathrm{~mm}$ ) en el 76,3\%. En este grupo, seis personas $(4,62 \%)$ habían presentado tuberculosis antes de los cinco años previos al estudio y 17 (10\%), la habían presentado en el grupo control. Estas diferencias no fueron estadísticamente significativas.

\section{Análisis de la efectividad de la profilaxis en los grupos de estudio}

Al final del estudio se diagnosticó un caso $(0,8 \%)$ de tuberculosis en el grupo de profilaxis y 10 casos $(5 \%)$ en el grupo control con un riesgo relativo (RR) de 0,15 IC 95\% entre 0,02 y 1,18 ( $p=0,07$ corrección de Yates). El caso de tuberculosis en el grupo expuesto a profilaxis, recibió el esquema de pirazinamida/rifampicina.

La proporción de incidencia de tuberculosis en el grupo control fue de 7,7 por 1.000 personas, en el grupo de profilaxis la incidencia disminuyó en $85 \%$ en comparación con el grupo de control que presentó una proporción de incidencia de 52,6 por 1.000 personas.

De los 11 casos de tuberculosis diagnosticados en el grupo de estudio, 10 pacientes recibían terapia antirretroviral, $R R=0,99$, IC95\% de 0,931,03, ( $p=0,38$, según corrección de Fisher).

La manifestación más frecuente de tuberculosis fue la forma pulmonar que ocurrió en siete personas, mientras la forma extrapulmonar se dio en cuatro casos. La única persona que presentó tuberculosis en el grupo que recibió profilaxis, tuvo una manifestación extrapulmonar (tuberculosis ganglionar) y había recibido el esquema pirazinamida/rifampicina.

\section{Efectos adversos de la profilaxis}

En total, 84 personas recibieron isoniacida y 47 , pirazinamida/rifampicina; en ellos la administración de la terapia profiláctica no produjo mayores efectos adversos, aunque sus manifestaciones fueron más frecuentes en el grupo que recibió el esquema de isoniacida, donde $8,3 \%$ de los tratados presentó dolores articulares, $7,1 \%$ cefalea y $4,8 \%$ epigastralgia. En este grupo, 3,6\% de los pacientes presentó coluria y $1,2 \%$ ictericia, efectos no relatados en el grupo que recibió pirazinamida/ rifampicina; en el grupo que recibió rifampicina/ pirazinamida la reacción más importante fue el dolor en epigastrio y el vómito en $8,5 \%$ de los pacientes (cuadro 2).

Cuadro 2. Distribución absoluta y porcentual de signos y síntomas reportados en pacientes que recibieron profilaxis para tuberculosis, Medellín, Colombia, 2002-2005.

\begin{tabular}{|c|c|c|c|c|c|}
\hline \multirow[b]{2}{*}{ Síntomas según la profilaxis } & \multicolumn{2}{|c|}{$\begin{array}{c}\text { Isoniacida } \\
(n=84)\end{array}$} & \multicolumn{2}{|c|}{$\begin{array}{l}\text { Pirazinamida/rifampicina } \\
\qquad(\mathrm{n}=47)\end{array}$} & \multirow[b]{2}{*}{ Valor de $\mathrm{P}$} \\
\hline & Número & $\%$ & Número & $\%$ & \\
\hline Parestesias en miembros inferiores & 1 & 1,2 & 1 & 2,1 & 0,69 \\
\hline Dolor para la marcha & 4 & 4,8 & 1 & 2,1 & 0,45 \\
\hline Ictericia & 1 & 1,2 & 0 & 0,0 & 0,45 \\
\hline Coluria & 3 & 3,6 & 0 & 0,0 & 0,08 \\
\hline Dolores articulares & 7 & 8,3 & 1 & 2,1 & 0,09 \\
\hline Cefalea que no cede con el analgésico & 6 & 7,1 & 1 & 2,1 & 0,15 \\
\hline Dolor epigástrico-vómito & 4 & 4,8 & 4 & 8,5 & 0,42 \\
\hline Otras complicaciones & 2 & 2,4 & 3 & 6,4 & 0,30 \\
\hline
\end{tabular}




\section{Egresos del estudio}

Se contabilizaron 20 egresos para el grupo expuesto a profilaxis y 14 para el grupo control (cuadro 3). En el grupo de profilaxis, seis pacientes se perdieron del estudio por cambios de afiliación de la institución aseguradora, mientras que en el grupo control ocurrieron cinco pérdidas $(p=0,11)$. Sólo tres pacientes egresaron del estudio por reacción adversa a la profilaxis. La mortalidad fue similar en ambos grupos ( $p=0,08)$; se observaron seis muertes en el grupo expuesto a la profilaxis y ocho en el grupo control. Las muertes ocurrieron por las siguientes causas: para el grupo que recibió profilaxis, dos presentaron sarcoma de Kaposi, otro meningitis y en los tres restantes no se obtuvo la causa. En el grupo control, uno presentó sarcoma de Kaposi, dos meningitis, una herida por arma de fuego, un accidente de tránsito y de los tres restantes no se obtuvo la causa de su fallecimiento.

\section{Análisis multivariado}

Con el fin de estimar la efectividad de la profilaxis, se ajustó por las diferencias encontradas en los niveles de CD4, CV y el uso de terapia antirretroviral (HAART), mediante un modelo de regresión logística (cuadro 4). Se encontró un nivel de efectividad del $80 \% \quad(1-0,20=0,80)$, independiente de las demás variables $(\mathrm{OR}=0,20$, IC95\% 0,02-1,34), sin diferencia estadísticamente significativa. El tener menos de 200 CD4 es factor de riesgo para desarrollar tuberculosis, en cambio es protector el tener una carga viral menor de 3.000 copias; el uso de HAART también mostró un efecto protector.

El tiempo de seguimiento promedio para el grupo que recibió profilaxis fue de 27,1 meses (IC 95\%: 26,9-27,2) y para el grupo control de 26,4 meses (IC 95\%: 25,7-26,9). Al analizar la supervivencia libre de tuberculosis durante el periodo de seguimiento, se encontró que fue significativamente mayor en el grupo que recibió quimioprofilaxis, el tiempo libre de tuberculosis en este grupo fue de dos años, mientras que a partir del tercer mes de seguimiento, se presentó el primer caso en el grupo control; la mediana del tiempo libre de tuberculosis para este grupo fue de 6,7 meses, las curvas del tiempo libre de tuberculosis fueron

Cuadro 3. Distribución absoluta y porcentual de las causas de egreso del estudio, Medellín, Colombia, 2002-2005.

\begin{tabular}{|c|c|c|c|c|c|}
\hline \multirow[b]{2}{*}{ Causas de egreso } & \multicolumn{2}{|c|}{$\begin{array}{l}\text { Grupo de profilaxis } \\
(n=131)\end{array}$} & \multicolumn{2}{|c|}{$\begin{array}{l}\text { Grupo control } \\
(n=200)\end{array}$} & \multirow[b]{2}{*}{ Valor de $\mathbf{P}$} \\
\hline & Número & $\%$ & Número & $\%$ & \\
\hline Pérdida & 6 & 4,6 & 5 & 2,5 & 0,33 \\
\hline Muerte & 6 & 4,6 & 8 & 4,0 & 0,08 \\
\hline Voluntad del paciente & 5 & 3,8 & 0 & - & - \\
\hline Reacción adversa a la profilaxis & 3 & 2,3 & 0 & - & - \\
\hline Sin dato & 0 & - & 1 & 0,5 & - \\
\hline Total & 20 & & 14 & & 0,02 \\
\hline
\end{tabular}

Cuadro 4. Nivel de efectividad de la profilaxis antibiótica contra la tuberculosis ajustado por otras variables del estudio mediante un modelo de regresión logística, Medellín, Colombia, 2002-2005.

\begin{tabular}{lrccrr}
\hline & B & Valor P & OR & \multicolumn{2}{c}{$\begin{array}{r}\text { IC95\% para exponente de beta* } \\
\text { Inferior }\end{array}$} \\
\hline Uso de profilaxis & $-1,77$ & 0,09 & 0,20 & 0,02 & 1,34 \\
CD4<200 & 0,81 & 0,33 & 2,30 & 0,45 & 11,30 \\
CV<3000 & $-1,70$ & 0,04 & 0,18 & 0,03 & 0,90 \\
Uso de terapia HAART & $-0,89$ & 0,41 & 0,41 & 0,04 & 3,47 \\
\hline
\end{tabular}

* Intervalo de confianza del $95 \%$ para el exponente de beta (OR) 
significativamente diferentes entre los grupos de estudio ( $L o g$ Rank test $\mathrm{p}=0,04$ ) (figura 1 ).

En relación con la incidencia de tuberculosis se examinó la asociación con factores que se han señalado en la literatura como pronósticos para el desarrollo de tuberculosis en pacientes con $\mathrm{VIH} /$ sida: la respuesta a la prueba de tuberculina (disponible sólo para el grupo de estudio), la velocidad de sedimentación globular, el número de linfocitos CD4 y el valor de la carga viral al inicio del estudio (cuadro 5). El único caso de tuberculosis ganglionar que se presentó en el grupo de profilaxis, tuvo una prueba de tuberculina negativa.

\section{Discusión}

El presente estudio fue de tipo observacional, lo que impone una limitación en la comparabilidad de los grupos de estudio, de hecho, los pacientes que aceptaron recibir profilaxis, provenían de estratos socioeconómicos más altos y tenían mejores condiciones inmunológicas, por lo cual se realizaron ajustes en el análisis de los datos.
Otra limitación proviene del seguimiento retrospectivo de la cohorte de control a partir de los registros médicos; sin embargo, para ambos grupos la fuente de información de seguimiento fue la misma, se anotaron las variables de estudio registradas en cada una de las visitas hechas por los pacientes sin presentarse diferencias en el número de visitas entre los grupos.

Podría esperarse un sesgo de vigilancia en el grupo que recibió profilaxis, al ser un grupo intervenido al que se le indagó mensualmente sobre el cumplimiento con la profilaxis y la presencia de los efectos secundarios; a pesar de ello, el número de visitas al programa fue la misma y sólo se registró un caso de tuberculosis en este grupo.

No se pudo establecer comparación entre los dos esquemas de profilaxis suministrados debido a la alerta de hepatopatías graves y muertes por la combinación entre inhibidores de proteasas y la pirazinamida/rifampicina (10), lo que redujo la posibilidad de captar un tamaño de muestra mayor.

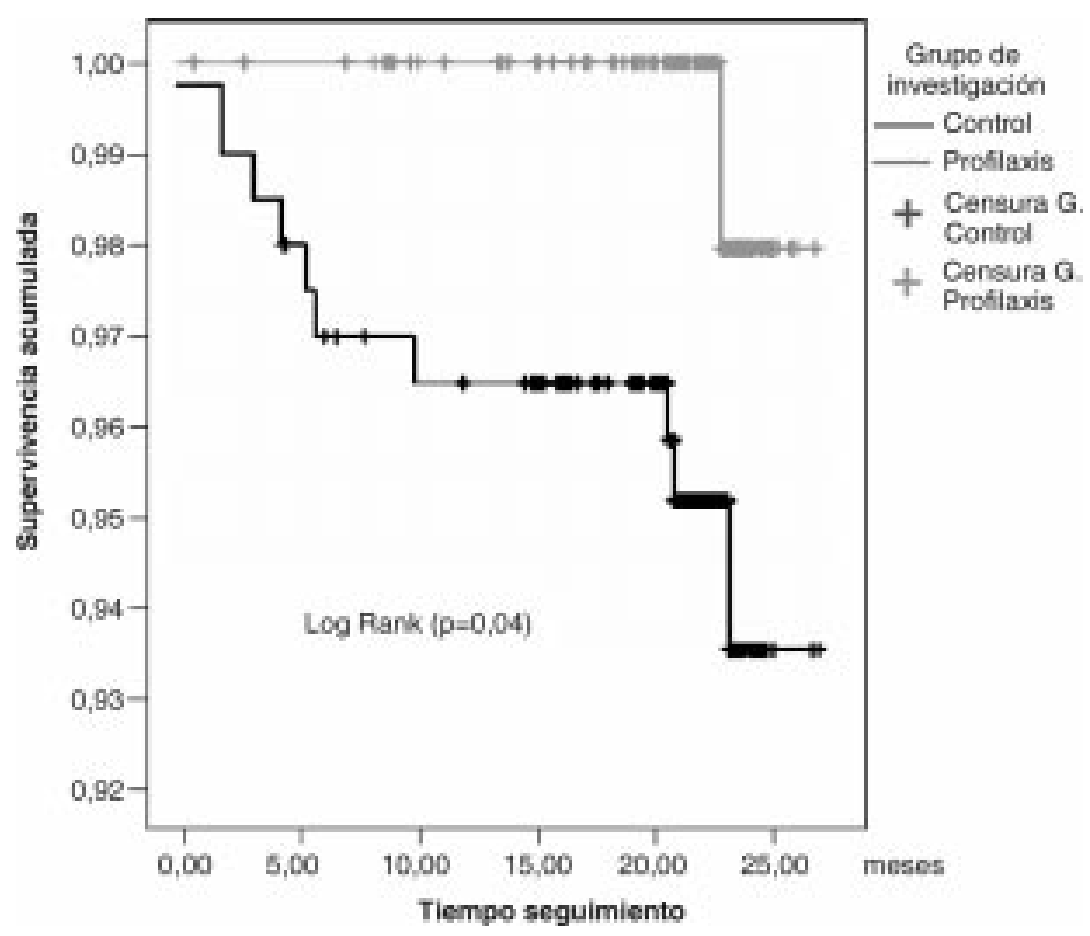

Figura 1. Supervivencia acumulada libre de tuberculosis en pacientes infectados por VIH según su exposición a profilaxis. Medellín, Colombia 2002-2005. 
Cuadro 5. Distribución absoluta y porcentual de las variables reportadas como predictoras de enfermedad tuberculosa, Medellín, Colombia, 2002-2005.

\begin{tabular}{|c|c|c|c|c|c|c|}
\hline & \multicolumn{4}{|c|}{ Tuberculosis } & \multirow[t]{2}{*}{$\mathbf{R} \mathbf{R}$} & \multirow[t]{2}{*}{ (IC95\%) } \\
\hline & $\begin{array}{c}\text { Sí } \\
(n=11)\end{array}$ & $\%$ & $\begin{array}{c}\text { No } \\
(n=320)\end{array}$ & $\%$ & & \\
\hline \multicolumn{7}{|l|}{ Velocidad de sedimentación globular } \\
\hline$<20$ & 2 & 18,2 & 109 & 34,1 & 1,00 & referencia \\
\hline$\geq 20$ & 9 & 81,8 & 211 & 65,9 & 2,27 & $(0,49-10,3)$ \\
\hline \multicolumn{7}{|l|}{ CD4 inicial } \\
\hline$<200$ & 2 & 18,2 & 64 & 20,0 & 1,00 & referencia \\
\hline Entre 200 y 350 & 5 & 45,5 & 58 & 18,1 & $0,78^{*}$ & $(0,17-3,51)$ \\
\hline$>350$ & 4 & 36,4 & 164 & 51,3 & & \\
\hline Sin dato & 0 & 0,0 & 34 & 10,6 & & \\
\hline \multicolumn{7}{|l|}{ Carga viral inicial } \\
\hline$<3.000$ & 2 & 18,2 & 167 & 52,2 & 1,00 & referencia \\
\hline Entre 3.000 y 10.000 & 2 & 18,2 & 18 & 5,6 & $1,56^{* *}$ & $(0,39-6,17)$ \\
\hline Entre 10.000 y 30.000 & 3 & 27,3 & 19 & 5,9 & & \\
\hline Entre 30.000 y 100.000 & 0 & 0,0 & 44 & 13,8 & & \\
\hline$>100.000$ & 3 & 27,3 & 30 & 9,4 & 7,68 & $(1,33-44,2)$ \\
\hline Sin dato & 1 & 9,1 & 42 & 13,1 & & \\
\hline
\end{tabular}

* Se agruparon los valores mayores de 200.

** Se agruparon los valores entre 3000 y 100000

El presente estudio aportó una protección del 80\%, la cual no presentó una diferencia estadísticamente significativa, posiblemente por limitaciones en el tamaño muestral; sin embargo, se considera que es un efecto protector importante que también se ha reportado en estudios realizados en poblaciones de alta prevalencia, como Madrid, Haití y Zambia, en donde los niveles de protección encontrados para la profilaxis han sido de $87 \%, 71 \%$ (estimados con base en los datos de los estudios) y de 40\%, respectivamente (12-14). Esta protección en el presente estudio no pudo ser evaluada en relación con el resultado de la prueba de tuberculina, ya que ésta no se aplicó en el grupo control; sin embargo, otros estudios han reportado que la protección persiste de manera independiente al resultado de esta prueba $(12,14-17)$.

Los 11 casos de tuberculosis diagnosticados, se presentaron en pacientes con recuentos menores de $500 \mathrm{CD} 4$, lo que es consistente con la inmunosupresión de estos pacientes en la presencia de la infección por VIH; esto sigue indicando la patogenicidad de $M$. tuberculosis y sustenta la recomendación del beneficio de la profilaxis en presencia de recuentos de CD4 por debajo de este nivel $(1,6,18)$.
Varias publicaciones han demostrado la efectividad de la terapia antirretroviral (HAART) para prevenir tuberculosis(2,19-20). Este estudio encontró que 10 de los 11 casos de tuberculosis se dieron en pacientes tratados con HAART. En relación con la terapia HAART, el estudio encontró problemas frecuentes en la administración oportuna y continua de la terapia antirretroviral. Esta condición hace pensar que el efecto encontrado en las dos cohortes observadas pudiera subestimar el efecto protector de la terapia HAART frente a la tuberculosis y que el suministro de antirretrovirales puede ser insuficiente para el control de la tuberculosis en países donde se presentan dificultades en el acceso regular a la terapia HAART y con alta prevalencia de la enfermedad, como en Colombia (21).

El diseño metodológico y los objetivos de este estudio no permiten demostrar que el suministro irregular de la terapia HAART podría explicar la presencia de tuberculosis, los resultados evidencian un efecto protector de esta terapia. En condiciones de administración irregular de la terapia HAART, la profilaxis para tuberculosis podría considerarse una medida preventiva de bajo costo y efectiva, para prevenir tanto la aparición 
de tuberculosis como de las complicaciones que acarrean la coinfección (20).

La profilaxis contra la tuberculosis ha demostrado su efectividad (22-24) y continúa teniendo una importancia elevada en la era HAART. Sin embargo, las guías recientes de los CDC no son concluyentes sobre la indicación de la profilaxis para los países en desarrollo con alta prevalencia de la enfermedad, y proponen que, en vista de la escasez de estudios que demuestren dicha efectividad, la decisión de usar la profilaxis debe ser individualizada (10).

En contraposición, la no realización de quimioprofilaxis universal en todos los pacientes infectados por el VIH presupone que deberían existir una indicaciones claras y precisas de a quién procurársela; sin embargo, no es así y es frecuente encontrarse con problemas derivados de la disponibilidad de las pruebas cutáneas de hipersensibilidad y de su interpretación. Las guías de atención para VIH que se tienen en Colombia, apoyan el uso de la profilaxis como herramienta para prevenir la aparición de tuberculosis (9); sin embargo, existen publicaciones que han generado controversia por la hepatotoxicidad que pudiera producir la profilaxis, principalmente la isonizacida, que pudiera favorecer la aparición de cepas de M. tuberculosis resistentes y también por las bajas tasas de cumplimiento con la profilaxis en este grupo específico $(25,26)$. En el presente estudio las reacciones adversas fueron reportadas en baja proporción y las mismas revirtieron en el momento en que se suspendió. De los 162 pacientes incluidos, los 131 que hicieron parte del análisis en el presente estudio completaron el periodo de profilaxis, según autorreporte de los pacientes a los investigadores en las llamadas telefónicas semanales y visitas mensuales que se realizaron durante el periodo de administración, lo que pudiera reflejar un cumplimiento adecuado. No se cuenta con la información de la sensibilidad de la micobacteria que afectó al paciente que presentó tuberculosis ganglionar en el grupo con profilaxis.

Estudios previos han demostrado cómo la profilaxis mejora la supervivencia de los pacientes coinfectados con VIH/tuberculosis, de hasta 111 meses (27); para el periodo comprendido durante el seguimiento de los pacientes que participaron en esta investigación, se registraron dos muertes menos en el grupo de profilaxis, pero no se analizó el efecto de la profilaxis en la supervivencia en esta cohorte.

El presente estudio refuerza la importancia de la profilaxis para tuberculosis en países con alta prevalencia de tuberculosis, como es el caso de Colombia, por lo que los autores recomendamos su utilización de acuerdo con la normatividad vigente para el país.

Los hallazgos sugieren también que en estos países el resultado de la prueba de tuberculina y los recuentos de CD4 pueden tener menor importancia en el momento de decidir la implementación de la profilaxis. El estudio deja también una discusión abierta sobre la efectividad de la profilaxis para prevenir tuberculosis en regiones con alta prevalencia de tuberculosis y en situaciones donde la cobertura con HAART no es adecuada. La duración de la profilaxis sigue siendo un aspecto por valorar ya que fue al final del segundo año de seguimiento que se presentó el caso de tuberculosis en el grupo que recibió esta medida, pero nuestro estudio no puede hacer recomendaciones sobre la protección de la profilaxis después del periodo de tiempo comprendido en el seguimiento.

\section{Conflicto de intereses}

Los autores manifestamos no tener ningún conflicto de intereses en el desarrollo ni en la divulgación de los resultados de la presente investigación.

\section{Financiación}

Colciencias proyecto No. 1115-04-11905, contrato RC No. 119-2002.

\section{Referencias}

1. Aukhtun OI, Golanov VS, Berezin NG, Kirbiat'eva EN. Clinical picture and treatment of tuberculosis in HIV-infected patients. Probl Tuberk. 2002;11:21-3.

2. Bowersox J. Short-course TB prophylaxis effective in HIV-infected individuals. NIAID AIDS Agenda. 1998; 8:4-5.

3. Charles M, Pape JW. Tuberculosis and HIV: Implications in the developing world. Curr HIV /AIDS Rep. 2006;3:139-44. 
4. Cahn P, Perez H, Ben G, Ochoa C. Tuberculosis and HIV: a partnership against the most vulnerable. J Int Assoc Physicians AIDS Care. 2003;2:106-23.

5. Aït KN, Enarson D, Billo N. The epidemiology of tuberculosis and of the resistance to antitubercular agents. Rev Mal Respir. 1997;14(Suppl.5):S8-18.

6. Blumberg HM, Leonard MK Jr, Jasmer RM. Update on the treatment of tuberculosis and latent tuberculosis infection. JAMA. 2005;293:2776-84.

7. Lawn SD, Bekker LG, Wood R. How effectively does HAART restore immune responses to Mycobacterium tuberculosis? Implications for tuberculosis control. AIDS. 2005;19:1113-24.

8. Elzi L, Schlegel M, Weber R, Hirschel B, Cavassini $\mathbf{M}$, Schmid $\mathbf{P}$, et al. Reducing tuberculosis incidence by tuberculin skin testing, preventive treatment, and antiretroviral therapy in an area of low tuberculosis transmission. Clin Infect Dis. 2007;44:94-102.

9. DíazGranados CA, Alvarez C, Prada G. Guía para el manejo de VIH/SIDA basada en la evidencia. Colombia. Bogotá: Fundación para la Investigación y el Desarrollo de la Salud y la Seguridad Social-FEDESALUD; 2005.

10. Center for Healthcare Environmental Management. CDC publishes flexible draft guidelines on tuberculosis control. Health Hazard Manage Monit. 2005;18:1-7.

11. Smith PG. Epidemiological methods to evaluate vaccine efficacy. Br Med Bull. 1988;44:679-90.

12. Cohen T, Lipsitch M, Walensky RP, Murray M. Beneficial and perverse effects of isoniazid preventive therapy for latent tuberculosis infection in HIV tuberculosis coinfected populations. Proc Natl Acad Sci USA. 2006;103:7042-7.

13. Pape JW, Jean SS, Ho JL, Hafner A, Johnson WD Jr. Effect of isoniazid prophylaxis on incidence of active tuberculosis and progression of HIV infection. Lancet. 1993;342:268-72

14. Moreno S, Baraia-Etxaburu J, Bouza E, Parras F, Perez-Tascon M, Miralles $\mathbf{P}$, et al. Risk for developing tuberculosis among anergic patients infected with HIV. Ann Intern Med. 1993;119:194-8.

15. Moreno S, Miralles P, Diaz MD, Baraia J, Padilla B, Berenguer $\mathbf{J}$, et al. Isoniazid preventive therapy in human immunodeficiency virus-infected persons. Longterm effect on development of tuberculosis and survival. Arch Intern Med. 1997;157:1729-34.
16. Moreno S, Podzamczer D, Blazquez R, Iribarren JA, Ferrer E, Reparaz J, et al. Treatment of tuberculosis in HIV-infected patients: safety and antiretroviral efficacy of the concomitant use of ritonavir and rifampin. AIDS. 2001;15:1185-7.

17. Woldehanna S, Volmink J. Treatment of latent tuberculosis infection in HIV infected persons. Cochrane Database Syst Rev. 2004;1:CD000171.

18. Pape JW. Tuberculosis and HIV in the Caribbean: approaches to diagnosis, treatment, and prophylaxis. Top HIV Med. 2004;12:144-9.

19. Gálvez Hermoso C, Blanco Quintana F, del Amo Valero J, Diez Ruiz-Navarro M, Soriano Vazquez V, Gonzalez Lahoz J. Effect of antiretroviral treatment and prophylaxis for opportunistic infections on survival of patients with AIDS. Rev Clin Esp. 2000;200:187-92.

20. Bandyopadhyay T, Murray H, Metersky ML. Costeffectiveness of tuberculosis prophylaxis after release from short-term correctional facilities. Chest. 2002;121:1771-5.

21. García I, Merchán A, Chaparro PE, López LE. Panorama de la coinfección tuberculosis/VIH en Bogotá, 2001. Biomédica. 2004;24 (Suppl.1):132-7.

22. Holmes CB, Wood R, Badri M, Zilber S, Wang B, Maartens G, et al. CD4 decline and incidence of opportunistic infections in Cape Town, South Africa: implications for prophylaxis and treatment. J Acquir Immune Defic Syndr. 2006,42:464-9.

23. Gordin F. Short-term tuberculosis prophylaxis is effective in persons with HIV. Am Fam Physician. 1998;58:948

24. Ronge R. What does tuberculosis prophylaxis bring in HIV infections? Dtsch Med Wochenschr. 1999;124:A8-9.

25. Gurumurthy P, Ramachandran G, Hemanth Kumar AK, Rajasekaran S, Padmapriyadarsini C, Swaminathan S, et al. Decreased bioavailability of rifampin and other antituberculosis drugs in patients with advanced human immunodeficiency virus disease. Antimicrob Agents Chemother. 2004;48:4473-5.

26. Lawn SD, Wood R. Tuberculosis control in South Africa -will HAART help? S Afr Med J. 2006;96:502-4.

27. Balcells ME, Thomas SL, Godfrey-Faussett $P$, Grant AD. Isoniazid preventive therapy and risk for resistant tuberculosis. Emerg Infect Dis. 2006;12:744-51. 